\title{
Calidad de vida relacionada a la salud bucal en niños peruanos con caries de infancia temprana severa
}

\author{
Oral health-related quality of life in peruvian \\ children with severe early childhood caries \\ Qualidade de vida relacionada à saúde bucal em crianças \\ peruanas com cárie grave na primeira infância \\ Evelyn R. Munayco-Pantoja ${ }^{1}$ ORCID: 0000-0003-0417-262X
Héctor Pereyra-Zaldívar² ORCID: 0000-0003-2449-4407
Marleny M. Cadillo-Ibarra³ ORCID: 0000-0003-4114-920X
}

\section{Resumen}

Objetivo: Evaluar la percepción parental sobre la calidad de vida relacionada a la salud bucal en niños con caries de infancia temprana severa de un instituto de salud peruano. Método: Se realizó un estudio observacional, analítico y transversal, en 200 niños de 3 a 5 años de edad, sin caries y con caries de infancia temprana severa (CIT-S) escogidos aleatoriamente. El instrumento aplicado por un encuestador calibrado fue la versión peruana del cuestionario ECOHIS (P-ECOHIS) y el índice ceo-s. La distribución normal fue determinada con Kolmogorov - Smirnov, también se aplicó la prueba estadística U de Mann-Whitney. Resultados: El puntaje total del ECOHIS es mayor en los niños con CIT-S comparado con los niños sin caries, con una diferencia estadísticamente significativa $(\mathrm{p}<0.05)$. Conclusión: La percepción parental sobre la calidad de vida relacionada a la salud bucal fue negativamente afectada por la caries de infancia temprana severa de los niños de un instituto de salud peruano.

Palabras clave: Caries dental, calidad de vida, odontología pediátrica, niño, preescolar.

\footnotetext{
Especialista en Odontopediatría. Facultad de Odontología. Universidad Nacional Mayor de San Marcos. Lima, Perú. evelyn.munayco@unmsm.edu.pe

2 Especialista en pediatría. Facultad de Medicina. Universidad Nacional Mayor de San Marcos. Lima, Perú. hpereyraz@unmsm.edu.pe

3 Especialista en Odontopediatría. Instituto Nacional de Salud del Niño. Lima, Perú. marle1328@hotmail.com
}

Fecha de recibido: 6/4/2020 - Fecha de aceptado: 12/6/2020 


\section{Abstract}

Objective: To evaluate the parental perception of the quality of life related to oral health in children with severe early childhood caries from a Peruvian health institute. Method: An observational, analytical and cross-sectional study was carried out in 200 children from 3 to 5 years of age, without caries and with randomly selected early childhood caries of severe childhood (CIT-S). The instrument applied by a calibrated surveyor was the Peruvian version of the ECOHIS questionnaire (P-ECOHIS) and the ceo-s index. The normal distribution was determined with Kolmogorov Smirnov, the Mann-Whitney U statistical test was also applied. Results: The total ECOHIS score is higher in children with CIT-S compared to children without caries, with a statistically significant difference ( $p$ $<0.05$ ). Conclusion: Parental perception of oral health-related quality of life was negatively affected by severe early childhood caries in children at a Peruvian health institute.

Keywords: Dental caries, quality of life, pediatric dentistry, child, preschool.

\section{Introducción y antecedentes}

La calidad de vida relacionada a la salud bucal (CVRSB), es un complejo multidimensional (funcional, psicosocial y económico) de dominios interrelacionados ${ }^{(1)}$ que representa la perspectiva subjetiva de la persona con respecto a síntomas y experiencias ${ }^{(2)}$. Es un término que forma parte de los indicadores socio dentales, definidos como la medida en que las condiciones orales influyen en el normal funcionamiento social y conducen a cambios importantes en las actividades cotidianas como incapacidad para trabajar o asistir a la escuela, etc ${ }^{(3)}$, siendo estos indicadores considerados

\section{Resumo}

Objetivo: Avaliar a percepção dos pais sobre a qualidade de vida relacionada à saúde bucal em crianças com cárie grave na primeira infância de um instituto de saúde peruano. Método: Foi realizado um estudo observacional, analítico e transversal em 200 crianças de 3 a 5 anos de idade, sem cárie e com cárie precoce da infância severa (CIT-S). O instrumento aplicado por um pesquisador calibrado foi a versão peruana do questionário ECOHIS (P-ECOHIS) e o índice ceo-s. A distribuição normal foi determinada com Kolmogorov - Smirnov, o teste estatístico Mann-Whitney U também foi aplicado. Resultados: O escore total do ECOHIS é maior em crianças com CIT-S em comparação com crianças sem cárie, com diferença estatisticamente significante $(p<0,05)$. Conclusão: A percepção dos pais sobre a qualidade de vida relacionada à saúde bucal foi afetada negativamente por cáries graves na primeira infância em crianças de um instituto de saúde peruano.

Palavras-chave: Cárie dentária, qualidade de vida, odontopediatría, criança, pré-escolar.

como complementos importantes de los indicadores clínicos ${ }^{(4)}$.

La caries de infancia temprana severa (CIT-S) está definida como cualquier signo de caries en superficies lisas en niños menores de 3 años; de 3 a 5 ańos, se caracteriza por presentar una o más superficies lisas cariadas, perdidas (debido a caries) u obturadas en los dientes primarios maxilares anteriores o con un ceo-s $\geq 4$ ( 3 años), $\geq 5$ (4 años) o $\geq 6$ (5 años) ${ }^{(5)}$. La etiología es compleja y multifactorial, al igual que la caries de infancia temprana $(\mathrm{CIT})^{(6)}$.

La prevalencia de la CIT-S se encuentra del $21 \%$ al $41.2 \%{ }^{(7-9)}$ en diferentes partes del mun- 
do. La prevalencia de CIT en Perú ya ha sido investigada anteriormente ${ }^{(10)}$, pero la prevalencia de CIT-S es desconocida en niños peruanos. Es importante investigarla porque los niños con CIT-S tienen alto riesgo de sufrir caries en dentición permanente ${ }^{(11)}$ y otras consecuencias clínicas, que pueden ser prevenidas si se detecta a tiempo.

La relación caries dental y la percepción parental sobre la CVRSB ha sido estudiada anteriormente, a nivel internacional ${ }^{(12-13)}$, latinoamericano ${ }^{(14-15)}$ y peruano ${ }^{(16)}$ encontrándose que la caries dental tiene un efecto negativo en la calidad de vida. Este efecto se puede explicar en el niño debido al dolor, las infecciones, mayor riesgo de hospitalización por complicaciones; en los padres, por los altos costos de tratamiento, noches sin dormir, días de trabajo perdidos $y$ angustia ${ }^{(17)}$. La relación tiende a fortalecerse a medida que empeora la gravedad de la enfermedad ${ }^{(18)}$ y son pocos los estudios que analizan esta relación en niños con CIT-S ${ }^{(19)}$.

En el Perú, la enfermedad que más afecta la salud oral en la población infantil es la caries dental, cuya prevalencia es del $59.1 \%$ en dentición decidua y $85.6 \%$ en dentición mixta ${ }^{(20)}$. Siendo una población con mayor riesgo de tener caries, debido a que las políticas de salud pública peruana no han dado prioridad a la salud oral ${ }^{(10)}$, es importante trabajar con ellos con un enfoque más integral, conjugar los componentes social y clínico, evaluando la calidad de vida y ver el problema como un todo, de esta manera habrá mayores probabilidades de prevenir la caries efectivamente. El objetivo del estudio fue evaluar la percepción parental sobre la calidad de vida relacionada a la salud bucal en niños con caries de infancia temprana severa de un instituto de salud peruano.

\section{Materiales y métodos}

\section{Población de estudio}

La investigación fue observacional, analítica y transversal, realizada en el Instituto Nacional de Salud del Niño (INSN) ubicado en Lima - Perú. Este instituto está especializado en la atención médica de niños y adolescentes. Es la primera institución pediátrica de referencia a nivel nacional. Los usuarios en su mayoría son de la capital $(83.24 \%)$ compuesta por población urbana $(75 \%)$, con mayor demanda de atención en niños menores de 9 años, donde solo el $49 \%$ son subvencionados por el seguro público gratuito ${ }^{(21)}$.

\section{Calibración del investigador}

La calibración consistió en dos pasos: El paso teórico implicó una discusión de los criterios del índice ceo-s a través del análisis de 30 fotografías a cargo de un especialista en odontología pediátrica (gold standard). El paso clínico se realizó en una institución educativa inicial, mediante el examen clínico de 30 niños, de 3 a 5 ańos, escogidos aleatoriamente por el gold standard. Los niños fueron evaluados 2 veces en un intervalo de 7 días. Se obtuvo una buena confiabilidad intraexaminador e interexaminador (Kappa de Cohen= 0.8 y 0.9 respectivamente). Luego se realizó un estudio piloto para evaluar la comprensión del cuestionario ECOHIS por parte de los cuidadores, donde no hubo malentendidos. Los nińos del estudio piloto y de la calibración no fueron incluidos en la muestra principal.

\section{Selección de la muestra}

El tamaño de la muestra fue determinado por conveniencia y escogida aleatoriamente (cada tercer niño de la lista de citados), estuvo conformada por 200 niños (100 sin caries y 100 con CIT-S), de 3 a 5 años que acudieron al departamento de odontología pediátrica en compañía de su cuidador durante el mes de octubre del 2019. Estos fueron invitados a participar del estudio según los siguientes criterios de inclusión, el cuidador debía ser el progenitor (padre o madre), el niño no debía padecer de ninguna enfermedad sistémica y debía contar con la historia clínica odontológica del instituto. 


\section{Recopilación de datos no clínicos}

Se utilizó la entrevista estructurada, realizada en la sala de espera del referido instituto. Se recolectó información sobre los datos demográficos del niño y del cuidador, así como la información de si contaba o no con algún tipo de seguro de salud.

El instrumento aplicado fue la versión peruana de la escala de impacto de salud oral en preescolares (P-ECOHIS) validada por López Ramos y col. ${ }^{(22)}$. Este evalúa el impacto o influencia de los problemas de salud oral relacionados a la calidad de vida en niños de tres a cinco años, tomando como referencia la percepción parental (23). Consta de 13 preguntas (items) divididas en dos partes principales: sección de impacto infantil (primera parte) y sección de impacto familiar (segunda parte). La sección de impacto infantil (SII), se compone de cuatro subescalas: síntoma infantil, función infantil, psicología infantil y auto-imagen del niño e interacción social. La sección de impacto familiar (SIF) contiene dos subescalas: angustia parental y función familiar. El cuestionario utiliza una escala Likert de cinco respuestas que van desde "nunca" a "muy a menudo" (equivalente a una puntuación de $0 \mathrm{a}$ 4 , respectivamente) ${ }^{(24)}$. Estas respuestas registran la frecuencia con la que ocurrió un evento durante la vida del niño. Cada elemento de la subescala se clasificó en aquellos sin impacto (puntaje 0 o 1) y aquellos con impacto (puntaje 2-4). Las opciones de respuesta "nunca" y "casi nunca" se consideraron que no tenían impacto en la CVRSB y las respuestas "ocasionalmente", "a menudo" y "muy a menudo" que sí tenían impacto ${ }^{(25)}$. La sexta alternativa de respuesta ("No sabe") no fue considerada en la investigación.

El puntaje total va desde cero a 52 calculada como una suma de las respuestas. Una mayor puntuación denota una peor percepción parental sobre la CVRSB, un efecto o influencia negativa ${ }^{(23)}$. Las diferentes secciones y puntajes se resumen en la Tabla 1. Esta etapa tuvo una duración aproximada de 10 minutos.

\section{Recopilación de datos clínicos}

Inmediatamente después de la entrevista, se recopilaron los datos clínicos, realizándose el diagnóstico con un espejo bucal, una sonda periodontal recomendada por la OMS (PCP 11.5B Hu Friedy ) y luz artificial. Se utilizó el índice ceo-s para discriminar entre los niños sin caries (ceo-s $=0$ ) y con CIT-S (ceo-s $\geq 4$, en niños de 3 años; ceo-s $\geq 5$, en niños de 4 años y ceo-s $\geq 6$, en niños de 5 años). Se considero como caries a las lesiones cariosas cavitadas independiente de la ubicación. Si el niño presentó placa bacteriana o restos de comida, se le indicó al cuidador realizarle la higiene respectiva antes de la evaluación. Teniendo esta etapa una duración aproximada de 5 minutos.

\section{Análisis estadístico}

Los datos recolectados fueron organizados en una matriz Excel (Microsoft Excel 2010). Se utilizó el paquete $\mathrm{R}$ versión 3.6.1 ( $\mathrm{R}$ Core Team de desarrollo, 2019). Se realizó un análisis estadístico descriptivo y una distribución de frecuencias de los datos demográficos, componentes, subescalas e ítems del ECOHIS en la muestra sin caries y con CIT-S. La edad del cuidador tuvo como punto de corte a la mediana. La distribución normal fue determinada con Kolmogorov - Smirnov, al no encontrar una distribución normal se aplicó una prueba no paramétrica. La prueba estadística U de MannWhitney con un nivel de confianza del 95\% determinó la diferencia estadística entre ambas muestras con respecto a las subescalas y puntaje total ECOHIS.

\section{Consideraciones éticas}

Este estudio fue aprobado por el Comité Institucional de Ética en Investigación del Instituto Nacional de Salud del niño (PI-08/19). Se obtuvo el consentimiento informado de todos los cuidadores incluidos en el estudio. 
Tabla 1. Resumen de las subescalas y puntajes del ECOHIS

\begin{tabular}{|c|c|c|c|}
\hline SECCIÓN DE IMPACTO INFANTIL (SII) & Subescalas & $\begin{array}{l}\text { Puntaje } \\
\text { mín. - máx. }\end{array}$ & Puntaje SII \\
\hline 1. ¿Con qué frecuencia su hijo ha tenido dolor en los dientes, boca o mandíbula? & Sintoma infantil & $0-4$ & \multirow{10}{*}{$0-36$} \\
\hline $\begin{array}{l}\text { ¿Con que frecuencia su hijo ha....................debido a problemas dentales o trata- } \\
\text { mientos odontológicos? }\end{array}$ & \multirow{5}{*}{ Función infantil } & \multirow{5}{*}{$0-16$} & \\
\hline 2. tenido dificultades para beber bebidas calientes o frías & & & \\
\hline 3. tenido dificultades para comer algunos alimentos & & & \\
\hline 4. tenido dificultades para pronunciar algunas palabras & & & \\
\hline 5. perdido días de asistencia a su actividad preescolar, de guardería o escuela & & & \\
\hline 6. tenido problemas para dormir & \multirow{2}{*}{ Psicología infantil } & \multirow{2}{*}{$0-8$} & \\
\hline 7. estado enojado o frustrado & & & \\
\hline 8. evitado sonreír & \multirow{2}{*}{$\begin{array}{l}\text { Autoimagen del niño/ } \\
\text { interacción social }\end{array}$} & \multirow{2}{*}{$0-8$} & \\
\hline 9. evitado hablar & & & \\
\hline SECCIÓN DE IMPACTO FAMILIAR (SIF) & Subescalas & $\begin{array}{c}\text { Puntaje } \\
\text { mín. - máx. }\end{array}$ & $\begin{array}{l}\text { Puntaje } \\
\text { SIF }\end{array}$ \\
\hline $\begin{array}{l}\text { ¿Con que frecuencia usted u otro miembro de su familia ................ } \\
\text { causa de los problemas dentales o tratamientos odontológicos de su hijo? }\end{array}$ & \multirow{3}{*}{ Angustia de los padres } & \multirow{3}{*}{$0-8$} & \multirow{5}{*}{$0-16$} \\
\hline 10. se ha sentido alterado / preocupado & & & \\
\hline 11. se ha sentido culpable & & & \\
\hline 12. ha ocupado tiempo de su trabajo/actividad & \multirow[b]{2}{*}{ Función familiar } & \multirow{3}{*}{$0-8$} & \\
\hline $\begin{array}{l}\text { 13. ¿Con que frecuencia los problemas dentales o tratamientos odontológicos de } \\
\text { su hijo han afectado a la economía en su familia/hogar? }\end{array}$ & & & \\
\hline PUNTAJE TOTAL ECOHIS & & & $0-52$ \\
\hline
\end{tabular}

\section{Resultados}

La edad promedio de los niños y de los cuidadores en la muestra sin caries y con CIT-S fue de $4.39 \pm 0.85 ; 32.83 \pm 6.82$ y $4.1 \pm 0.72$; $31.4 \pm 5.81$ respectivamente. La mayoría de los participantes con CIT-S fueron niños de sexo masculino $(55 \%)$ y en mayor proporción estuvieron acompañados por sus madres (89\%). El $65 \%$ contaba con un seguro de salud. El promedio de los valores del ceo-s en los niños de 3,4 y 5 ańos fue de $6.4 \pm 2.79,7.12 \pm 3.48$ y $9.05 \pm 3.56$ respectivamente. Los datos demográficos correspondientes a los niños sin caries se observan en la Tabla 2.

En la Tabla 3, la sección infantil y familiar de los niños sin caries tuvieron valores más altos en las respuestas "nunca" y "casi nunca". Estos niños obtuvieron un 98\%, 99\% y $100 \%$, en la sección infantil, como respuesta la alternativa "nunca" en los ítems "dificultad para pronunciar palabras", "ha evitado sonreír", "ha evitado hablar", "dificultad para beber" y "si el niño se ha sentido enojado o frustrado". En la sección familiar, han presentado puntajes bajos en la respuesta "nunca" para los ítems "se ha alterado o preocupado", "se ha sentido culpable" y "ha ocupado tiempo de su trabajo o actividad". Además, los niños sin caries obtuvieron valores de 0 en la respuesta "a menudo" para todos los ítems de la sección infantil.

Se observa en la Tabla 4, que el puntaje total del ECOHIS fue mayor en los niños con CIT-S comparado con los niños sin caries. La sección familiar fue el más impactado por tener los valores más altos, siendo los ítems "se ha alterado o preocupado", "se ha sentido culpable" y "ha ocupado tiempo de su trabajo o actividad" los 
Tabla 2. Características demográficas de los participantes del estudio

\begin{tabular}{|c|c|c|c|}
\hline Características demográficas & & Muestra sin caries & Muestra con caries \\
\hline Género cuidador & Femenino & $96 \%$ & $89 \%$ \\
\hline \multirow{2}{*}{ Género del niño } & Masculino & $4 \%$ & $11 \%$ \\
\hline \multirow{2}{*}{ Edad del cuidador } & Femenino & $45 \%$ & $45 \%$ \\
\hline & Masculino & $55 \%$ & $55 \%$ \\
\hline \multirow{2}{*}{ Edad del niño } & $20-31$ & $42 \%$ & $52 \%$ \\
\hline & $32-43$ & $58 \%$ & $48 \%$ \\
\hline & 3 años & $24 \%$ & $21 \%$ \\
\hline \multirow{2}{*}{ Seguro de salud } & 4 años & $63 \%$ & $48 \%$ \\
\hline & 5 años & $13 \%$ & $31 \%$ \\
\hline & Presente & $58 \%$ & $65 \%$ \\
\hline
\end{tabular}

Tabla 3. Distribución de las respuestas del ECOHIS según subescalas e ítems en niños peruanos sin caries y con CIT-S $(\mathbf{n}=200)$

\begin{tabular}{|c|c|c|c|c|c|c|c|c|c|c|}
\hline \multirow[b]{2}{*}{ Sección, ítems } & \multicolumn{2}{|c|}{ Nunca } & \multicolumn{2}{|c|}{ Casi nunca } & \multicolumn{2}{|c|}{ Ocasionalmente } & \multicolumn{2}{|c|}{ A menudo } & \multicolumn{2}{|c|}{ Muy a menudo } \\
\hline & $\mathbf{a}$ & b & $\mathbf{a}$ & b & a & b & a & b & a & b \\
\hline \multicolumn{11}{|l|}{ Infantil } \\
\hline Dolor en dientes, boca o mandíbula & 83 & 20 & 7 & 38 & 6 & 22 & 4 & 7 & 0 & 13 \\
\hline Dificultad para beber & 98 & 58 & 1 & 20 & 1 & 10 & 0 & 6 & 0 & 6 \\
\hline Dificultad para comer & 85 & 31 & 6 & 22 & 6 & 26 & 3 & 11 & 0 & 10 \\
\hline Dificultad para pronunciar palabras & 100 & 77 & 0 & 12 & 0 & 8 & 0 & 2 & 0 & 1 \\
\hline $\begin{array}{l}\text { Perdida de asistencia a escuela, } \\
\text { guardería }\end{array}$ & 77 & 18 & 8 & 25 & 4 & 20 & 11 & 19 & 0 & 18 \\
\hline Problemas para dormir & 94 & 49 & 2 & 31 & 1 & 11 & 3 & 6 & 0 & 3 \\
\hline Se ha sentido enojado o frustrado & 98 & 34 & 2 & 20 & 0 & 26 & 0 & 14 & 0 & 6 \\
\hline Ha evitado sonreír & 99 & 73 & 1 & 20 & 0 & 0 & 0 & 5 & 0 & 2 \\
\hline Ha evitado hablar & 99 & 77 & 1 & 17 & 0 & 5 & 0 & 1 & 0 & 0 \\
\hline \multicolumn{11}{|l|}{ Familiar } \\
\hline Se ha alterado o preocupado & 4 & 7 & 17 & 6 & 28 & 20 & 15 & 28 & 36 & 39 \\
\hline Se ha sentido culpable & 4 & 6 & 16 & 12 & 29 & 20 & 15 & 25 & 36 & 37 \\
\hline $\begin{array}{l}\text { Ha ocupado tiempo de su trabajo } 0 \\
\text { actividad }\end{array}$ & 6 & 3 & 22 & 14 & 25 & 19 & 20 & 30 & 27 & 34 \\
\hline Economía familiar afectada & 17 & 8 & 30 & 28 & 35 & 26 & 7 & 21 & 11 & 17 \\
\hline
\end{tabular}

$\mathrm{a}=$ niños $\sin$ caries, $\mathrm{b}=$ niños con CIT-S, valores en porcentaje 
Tabla 4. Distribución de las puntuaciones del ECOHIS con respecto al impacto infantil y familiar en nińos peruanos sin caries y con CIT-S ( $\mathbf{n}=\mathbf{2 0 0})$

\begin{tabular}{|c|c|c|c|c|c|c|}
\hline & \multicolumn{2}{|c|}{ Media \pm DS } & \multicolumn{2}{|c|}{$\begin{array}{c}\text { Mínimo - máximo valor } \\
\text { obtenido }\end{array}$} & \multicolumn{2}{|c|}{ Impacto (\%) } \\
\hline & a & b & a & b & $\mathbf{a}$ & b \\
\hline Impacto infantil & $1.42 \pm 2.43$ & $9.1 \pm 6.58$ & $0-11$ & $0-29$ & & \\
\hline Dolor en dientes, boca o mandíbula & $0.31 \pm 0.76$ & $1.55 \pm 1.26$ & $0-3$ & $0-4$ & 10 & 42 \\
\hline Dificultad para beber & $0.03 \pm 0.22$ & $0.82 \pm 1.2$ & $0-2$ & $0-4$ & 1 & 22 \\
\hline Dificultad para comer & $0.27 \pm 0.71$ & $1.47 \pm 1.31$ & $0-3$ & $0-4$ & 9 & 47 \\
\hline Dificultad para pronunciar palabras & 0 & $0.38 \pm 0.81$ & 0 & $0-4$ & 0 & 11 \\
\hline Perdida de asistencia a escuela, guardería & $0.64 \pm 1.34$ & $1.94 \pm 1.38$ & $0-4$ & $0-4$ & 15 & 57 \\
\hline Problemas para dormir & $0.13 \pm 0.56$ & $0.83 \pm 1.04$ & $0-3$ & $0-4$ & 4 & 20 \\
\hline Se ha sentido enojado o frustrado & $0.02 \pm 0.14$ & $1.38 \pm 1.25$ & $0-1$ & $0-4$ & 0 & 46 \\
\hline Ha evitado sonreír & $0.01 \pm 0.1$ & $0.43 \pm 0.89$ & $0-1$ & $0-4$ & 0 & 7 \\
\hline Ha evitado hablar & $0.01 \pm 0.1$ & $0.3 \pm 0.61$ & $0-1$ & $0-3$ & 0 & 6 \\
\hline Impacto familiar & $9.3 \pm 4.19$ & $10.5 \pm 3.39$ & $0-16$ & $2-16$ & & \\
\hline Se ha alterado o preocupado & $2.62 \pm 1.25$ & $2.86 \pm 1.21$ & $0-4$ & $0-4$ & 79 & 87 \\
\hline Se ha sentido culpable & $2.63 \pm 1.24$ & $2.75 \pm 1.24$ & $0-4$ & $0-4$ & 70 & 82 \\
\hline Ha ocupado tiempo de su trabajo o actividad & $2.4 \pm 1.26$ & $2.78 \pm 1.15$ & $0-4$ & $0-4$ & 72 & 83 \\
\hline Economía familiar afectada & $1.65 \pm 1.78$ & $2.11 \pm 1.22$ & $0-4$ & $0-4$ & 53 & 64 \\
\hline Puntaje total ECOHIS & $10.72 \pm 5.58$ & $19.6 \pm 8.56$ & $0-27$ & $5-45$ & & \\
\hline
\end{tabular}

DS = Desviación estándar, $\mathrm{a}=$ niños sin caries, $\mathrm{b}=$ niños con CIT-S

Tabla 5. Descripción de medianas y rango intercuartil de cada subescala y puntaje total ECOHIS en nińos peruanos sin caries y con CIT-S $(\mathbf{n}=200)$

\begin{tabular}{|l|c|c|c|}
\hline Subescalas & Sin caries & Con CIT-S & valor p \\
\hline Síntoma infantil & $0(0)$ & $1(1)$ & $<0.001^{*}$ \\
\hline Función infantil & $0(1)$ & $4(5)$ & $<0.001^{*}$ \\
\hline Psicología infantil & $0(0)$ & $2(3)$ & $<0.001^{*}$ \\
\hline Autoimagen/interacción social & $0(0)$ & $0(1)$ & $<0.001^{*}$ \\
\hline Angustia de los padres & $6(4)$ & $6(4)$ & 0.41 \\
\hline Función familiar & $4(4)$ & $5(2)$ & $<0.01^{*}$ \\
\hline Puntaje total ECOHIS & $11(8.25)$ & $18(12)$ & $<0.001^{*}$ \\
\hline
\end{tabular}

$*_{\mathrm{p}}<0.05$ - estadísticamente significativo, prueba U de Mann-Whitney

más impactados. Los menos impactados por la CIT-S fueron los ítems "ha evitado sonreír" y "ha evitado hablar", con un porcentaje de 7 y 6 respectivamente, pertenecientes a la sección infantil.
La Tabla 5, se observa la diferencia estadísticamente significativa $(\mathrm{p}<0.05)$ entre el impacto de las subescalas y puntaje total del ECOHIS entre los niños sin caries y con CIT-S, excepto en la subescala angustia de los padres. 


\section{Discusión}

La razón principal para la prevención y tratamiento de la caries en la dentición primaria es para evitar el dolor y la infección, ya que esto ocasiona un impacto considerable en el bienestar psicosocial de los niños y puede afectar el desempeño de las actividades de la vida diaria (26). La salud de los niños, afecta económica y emocionalmente a todos los integrantes de la familia, convirtiendo a la caries dental en un problema familiar y social, más aún, siendo Perú uno de los países con mayor prevalencia de caries sin tratar en la región ${ }^{(10)}$, se convierte en un problema de salud pública de interés nacional.

Se ha demostrado que la caries de infancia temprana (CIT) tiene un impacto negativo en la percepción parental sobre la CVRSB de los niños y sus padres/cuidadores ${ }^{(27-30)}$. En contraparte, en el estudio publicado por Ramos-Jorge y col. ${ }^{(31)}$, el 52,8\% de los padres/ cuidadores considera que la caries no impacta en la calidad de vida, donde el 60.6\% de niños tiene caries severa. En la presente investigación, los cuidadores reportaron que la CIT$S$ impactaba negativamente en la percepción parental sobre la CVRSB (todos los ítems del ECOHIS tienen porcentajes altos excepto en los ítems "ha evitado sonreír" y "ha evitado hablar"), similar resultado fue encontrado en el continente asiático ${ }^{(19,6)}$. Por lo que se deduce que la CIT-S, es la que causa mayores consecuencias en los niños y las familias. Aun así, la mayoría de las investigaciones consultadas sobre caries en preescolares se centra en CIT, siendo esto una de las limitaciones del estudio.

El puntaje medio del ECOHIS para los niños con CIT-S fue de $19.6 \pm 8.56$, el cual fue mayor comparado con los niños sin caries, similar resultado fue encontrado por Lai y col. (19) $(14.3 \pm 7.9)$; estos valores son próximos a pesar que las poblaciones comparadas pertenecen a diferentes continentes. Cabe destacar que los puntajes obtenidos por la CIT son menores $(9.21 ; 3.1 \pm 5.1 ; 2.95)$ según Abanto y col. ${ }^{(32)}$, Li MY y col. ${ }^{(33)}$ y Martins-Junior y col. ${ }^{(30)}$, respectivamente, comparados con los obtenidos en esta investigación. Es importante conocer el puntaje total obtenido del ECOHIS, ya que, a mayor puntaje, la CVRSB está más impactada. En este estudio, ambos componentes del ECOHIS están afectados por la CIT-S, en mayor medida el componente familiar, similar resultado encontrado por Lai y col. ${ }^{(19)}$, en contraposición con lo encontrado por Scarpelli y col. ${ }^{(27)}$, Gomes y col. ${ }^{(29)}$; y Martins-Júnior y col. ${ }^{(30)}$ donde el componente infantil es el más impactado por la CIT.

Dentro del componente infantil, lo que más destaca es la perdida de días de asistencia a la escuela (57\%), tópico estudiado por Ruff y col. ${ }^{(34)}$, quienes mostraron que los niños con necesidades dentales no satisfechas pueden estar más tiempo ausentes debido a que deben acudir al dentista para recibir tratamiento y el dolor hace que tengan dificultades para atender las clases, afectando su rendimiento escolar. Las preguntas relacionadas al dolor y la dificultad para comer han sido reportadas con mayor frecuencia ${ }^{(27,30)}$, aunque, más de la mitad de los padres, de este estudio, respondieron que no impactaba en la percepción parental sobre la CVRSB. Aun así, el dolor producido por la CIT podría afectar la nutrición infantil; ya que varios estudios han encontrado que, por cada unidad de aumento en la frecuencia del dolor dental, los niños tenían mayores probabilidades de tener bajo peso ${ }^{(35)}$, lo cual está asociado a la desnutrición (36).

$\mathrm{Al}$ evaluar el componente familiar, los resultados son semejantes a los encontrados por Lai y col. ${ }^{(19)}$; se evidencia la culpa de los padres (82\%), estudiada anteriormente por Carvalho y col. ${ }^{(37-38)}$; y Gomes y col. ${ }^{(39)}$. Estos investigadores encuentran que un tercio de ellos se sienten culpables, lo que provoca una disminución en la percepción parental sobre la CVRSB de las 
familias y que está asociada con el pensamiento de que el niño tiene problemas en los dientes y que esto podría haberse evitado. La subescala angustia de los padres no presentó una diferencia en los puntajes relacionados a los niños con CIT-S y los niños sin caries, además los puntajes de esta subescala son más altos que los publicados por Li y col. en el año $2015^{(33)}$.

Otro ítem importante, dentro del componente familiar, es el tiempo que ocupan los padres acompañando a sus hijos a los tratamientos dentales (83\%), estudiado por Ribeiro y col. (40) como ausencia al trabajo. Estos investigadores concluyen que está asociado con el dolor dental, lo cual afecta el funcionamiento familiar. Con respecto a la afectación económica (64\%) Ribeiro y col. ${ }^{(41)}$ señala que los padres experimentaron un impacto financiero debido a la búsqueda tardía de tratamiento, principalmente por la presencia de dolor y complicaciones clínicas.

Todos estos efectos negativos de la CIT-S comparados con niños sin caries, sobre la CVRSB, encontrados en el presente estudio deberían tenerse en cuenta para implementar mejores políticas públicas enfocadas en adoptar medidas preventivas en evitar la aparición de caries, fortaleciendo las estrategias de atención primaria en salud en el primer nivel de atención e instaurarlas como una prioridad dentro de las medidas de salud pública peruana, que en la actualidad no han sido efectivas controlando la enfermedad. Se recomienda, que las futuras investigaciones sobre el tema, incluyan una muestra mayor para que los resultados se puedan extrapolar a la población.

\section{Conclusión}

La percepción parental sobre la calidad de vida relacionada a la salud bucal fue negativamente afectada por la caries de infancia temprana severa de los niños de un instituto de salud peruano.

\section{Contribución de autoría}

1. Concepción y diseño del estudio

2. Adquisición de datos

3. Análisis de datos

4. Discusión de los resultados

5. Redacción del manuscrito

6. Aprobación de la versión final del manuscrito

ERMP ha contribuido en 1, 2, 3, 4 y 5.

HPZ ha contribuido en 3, 4.

MMCI ha contribuido en 2, 6 .

\section{Referencias}

1. Gift HC, Atchison KA, Dayton CM. Conceptualizing oral health and oral health-related quality of life. Soc Sci Med. 1997; 44 (5): 601-608.

2. Sischo L, Broder HL. Oral health-related quality of life: what, why, how, and future implications. J Dent Res. 2011; 90 (11): 1264-1270.

3. Locker D, Allen PF. Developing short-form measures of oral health-related quality of life. J Public Health Dent. 2002 Winter; 62 (1): 1320.

4. Maizels J, Maizels A, Sheiham A. Dental disease and health behaviour: the development of an interactional model. Community Dent Health. 1991; 8 (4): 311-21.

5. AAPD. Definition of early childhood caries (ECC): Classifications, consequences, and preventive strategies. Pediatr Dent Ref Man, 201213; 34: 12-3.

6. Mansoori S, Mehta A, Ansari MI. Factors associated with Oral Health Related Quality of Life of children with severe -Early Childhood Caries. J Oral Biol Craniofac Res. 2019; 9 (3): 222-225.

7. Li Y, Wulaerhan J, Liu Y, Abudureyimu A, Zhao J. Prevalence of severe early childhood caries and associated socioeconomic and behavioral factors in Xinjiang, China: a cross-sectional study. BMC Oral Health. 2017; 17 (1): 144.

8. G Mangla R, Kapur R, Dhindsa A, Madan M. Prevalence and associated Risk Factors of Severe Early Childhood Caries in 12- to 36-monthold Children of Sirmaur District, Himachal 
Pradesh, India. Int J Clin Pediatr Dent. 2017; 10 (2): 183-187.

9. Schroth RJ, Halchuk S, Star L. Prevalence and risk factors of caregiver reported Severe Early Childhood Caries in Manitoba First Nations children: results from the RHS Phase 2 (20082010). Int J Circumpolar Health. 2013; 72.

10. Castillo JL, Palma C, Cabrera-Matta A. Early Childhood Caries in Peru. Front Public Health. 2019; 7: 337.

11. Xie YY, Cheng ML, Xu MR, Si Y, Xu T. Costeffectiveness Analysis of Comprehensive Oral Health Care for Severe Early Childhood Caries in Urban Beijing, China. Chin J Dent Res. 2019; 22 (1): 45-50.

12. Duangthip D, Gao SS, Chen KJ, Lo ECM, $\mathrm{Chu} \mathrm{CH}$. Oral health-related quality of life and caries experience of Hong Kong preschool children. Int Dent J. 2019; 70 (2): 100-107

13. Sakaryali D, Bani M, Cinar C, Alacam A. Evaluation of the impact of early childhood caries, traumatic dental injury, and malocclusion on oral health-Related quality of life for Turkish preschool children and families. Niger J Clin Pract. 2019; 22 (6): 817-823.

14. Nóbrega AVD, Moura LFAD, Andrade NS, Lima CCB, Dourado DG, Lima MDM. Impact of dental caries on the quality of life of preschoolers measured by PedsQL questionnaire. Cien Saude Colet. 2019; 24 (11): 4031-4042.

15. Díaz S, Mondol M, Peñate A, Puerta G, Boneckér M, Martins Paiva S, et al. Parental perceptions of impact of oral disorders on Colombian preschoolers oral health-related quality of life. Acta Odontol Latinoam. 2018; 31 (1): 23-31.

16. Pesaressi E, Villena RS, Frencken JE. Dental caries and oral health-related quality of life of 3 -year-olds living in Lima, Peru. Int J Paediatr Dent. 2020; 30 (1): 57-65.

17. BaniHani A, Deery C, Toumba J, Munyombwe T, Duggal M. The impact of dental caries and its treatment by conventional or biological approaches on the oral health-related quality of life of children and carers. Int J Paediatr Dent. 2018; 28 (2): 266-276.

18. Nora ÂD, da Silva Rodrigues C, de Oliveira Rocha R, Soares FZM, Minatel Braga M, Lenzi TL. Is Caries Associated with Negative Impact on Oral Health-Related Quality of Life of Pre-school Children? A Systematic Review and Meta-Analysis. Pediatr Dent. 2018; 40 (7): 403-411.

19. Lai SHF, Wong MLW, Wong HM, McGrath CPJ, Yiu CKY. Factors influencing the oral health-related quality of life among children with severe early childhood caries in Hong Kong. Int J Dent Hyg. 2019; 17 (4): 350-358.

20. Perú.Ministerio de salud [Internet]. Guía de práctica clínica para la prevención, diagnóstico y tratamiento de la caries dental en niñas y niños: Guía técnica [citado el 14 de noviembre del 2019]. Disponible en: http: //bvs.minsa. gob.pe/local/MINSA/4195.pdf.

21. Perú. Instituto Nacional de Salud del Niño [Internet]. Análisis Situacional de los Servicios de Salud del Instituto Nacional de salud del Niño - 2018 [citado el 20 de diciembre del 2019]. Disponible en: http: //www.insn.gob.pe/publica-ciones/asis.

22. López Ramos RP, García Rupaya CR, VillenaSarmiento R, Bordoni NE. Cross cultural adaptation and validation of the Early Childhood Health Impact Scale (ECOHIS) in Peruvian preschoolers. Acta Odontol Latinoam. 2013; 26 (2): 60-7.

23. Pahel BT, Rozier RG, Slade GD.Parental perceptions of children's oral health: the Early Childhood Oral Health Impact Scale (ECOHIS). Health Qual Life Outcomes. 2007; 5: 6.

24. Martins-Júnior PA, Ramos-Jorge J, Paiva SM, Marques LS, Ramos-Jorge ML. Validations of the Brazilian version of the Early Childhood Oral Health Impact Scale (ECOHIS). Cad Saude Pública. 2012; 28 (2): 367-74.

25. Arrow P, Klobas E. Evaluation of the Early Childhood Oral Health Impact Scale in an Australian preschool child population. Aust Dent J. 2015; 60 (3): 375-81.

26. Ferraz NK, Nogueira LC, Pinheiro ML, Marques LS, Ramos-Jorge ML, Ramos-Jorge J. Clinical consequences of untreated dental caries and toothache in preschool children. Pediatr Dent. 2014; 36 (5): 389-92.

27. Scarpelli AC, Paiva SM, Viegas CM, Carvalho AC, Ferreira FM, Pordeus IA. Oral health-related quality of life among Brazilian preschool children. Community Dent Oral Epidemiol. 2013; 41(4): 336-44.

28. Abanto J, Paiva SM, Raggio DP, Celiberti P, Aldrigui JM, Bönecker M. The impact of dental 
caries and trauma in children on family quality of life. Community Dent Oral Epidemiol. 2012; 40 (4): 323-31.

29. Gomes MC, Pinto-Sarmento TC, Costa EM, Martins CC, Granville-Garcia AF, Paiva SM. Impact of oral health conditions on the quality of life of preschool children and their families: a cross-sectional study. Health Qual Life Outcomes. 2014; 12: 55.

30. Martins-Júnior PA, Vieira-Andrade RG, Corrêa-Faria P, Oliveira-Ferreira F, Marques LS, Ramos-Jorge ML. Impact of early childhood caries on the oral health-related quality of life of preschool children and their parents. Caries Res. 2013; 47 (3): 211-8.

31. Ramos-Jorge J, Pordeus IA, Ramos-Jorge ML, Marques LS, Paiva SM. Impact of untreated dental caries on quality of life of preschool children: different stages and activity. Community Dent Oral Epidemiol. 2014; 42 (4): 311-22.

32. Abanto J, Carvalho TS, Mendes FM, Wanderley MT, Bönecker M, Raggio DP. Impact of oral diseases and disorders on oral health-related quality of life of preschool children. Community Dent Oral Epidemiol. 2011; 39 (2): 105-114.

33. Li MY, Zhi QH, Zhou Y, Qiu RM, Lin HC. Impact of early childhood caries on oral healthrelated quality of life of preschool children. Eur J Paediatr Dent. 2015; 16 (1): 65-72.

34. Ruff RR, Senthi S, Susser SR, Tsutsui A. Oral health, academic performance, and school absenteeism in children and adolescents: A systematic review and meta-analysis. J Am Dent Assoc. 2019; 150 (2): 111-121.
35. So M, Ellenikiotis YA, Husby HM, Paz CL, Seymour B, Sokal-Gutierrez K. Early Childhood Dental Caries, Mouth Pain, and Malnutrition in the Ecuadorian Amazon Region. Int J Environ Res Public Health. 2017; 14 (5).

36. Janakiram C, Antony B, Joseph J. Association of Undernutrition and Early Childhood Dental Caries. Indian Pediatr. 2018; 55 (8): 683-685.

37. Carvalho TS, Abanto J, Pinheiro ECM, Lussi A, Bönecker M. Early childhood caries and psychological perceptions on child's oral health increase the feeling of guilt in parents: an epidemiological survey. Int J Paediatr Dent. 2018; 28 (1): 23-32.

38. Carvalho TS, Abanto J, Mendes FM, Raggio DP, Bonecker M. Association between parental guilt and oral health problems in preschool children. Braz Oral Res 2012; 26: 557-563.

39. Gomes MC, Clementino MA, Pinto-Sarmento TC, Martins CC, Granville-Garcia AF, Paiva SM. Association between parental guilt and oral health problems in preschool children: a hierarchical approach. BMC Public Health 2014; 14: 854.

40. Ribeiro GL, Gomes MC, de Lima KC, Martins CC, Paiva SM, Granville-Garcia AF. Work absenteeism by parents because of oral conditions in preschool children. Int Dent J. 2015; 65 (6): 331-7.

41. Ribeiro GL, Gomes MC, de Lima KC, Martins CC, Paiva SM, Granville-Garcia AF. The potential financial impact of oral health problems in the families of preschool children. Cien Saude Colet. 2016; 21 (4): 1217-26.

Evelyn R. Munayco-Pantoja: evelyn.munayco@unmsm.edu.pe 